\title{
Comparative cross-sectional study of empathy among first year and final year medical students in Jimma University, Ethiopia: Steady state of the heart and opening of the eyes
}

Sandra Dehning ${ }^{1 \dagger}$, Eshetu Girma ${ }^{2 *}$, Sarah Gasperi ${ }^{1 \dagger}$, Sebastian Meyer ${ }^{1 \dagger}$, Markos Tesfaye ${ }^{3+}$ and Matthias Siebeck ${ }^{4 \dagger}$

\begin{abstract}
Background: There is general consent that empathy is crucial for the physician-patient relationship and thus an important issue in medical education. This comparative study was designed to examine the differences in empathy between first year and final year medical students in Jimma University, Ethiopia.

Methods: A comparative cross-sectional study among 131 first year and 106 final year medical students was conducted in Jimma University, Ethiopia on academic year 2010/11. The study subjects were selected using simple random sampling technique from the list of the students. Study participation was voluntary. The Balanced Emotional Empathy Scale (BEES) was used for the detection of "heart-reading", i.e. emotional empathy and the Reading the Mind in the Eyes test (RME-R test) to evaluate "mind-reading", i.e. cognitive empathy. We performed t-test to compare the mean difference in empathy and RME-R scores between the two groups of students. A linear regression was computed to identify potential factors influencing the BEES and RME-R.
\end{abstract}

Results: Out of the total 237 students, 207 (87.3\%) were males. The mean age of first year and final year students was $19.3 \pm 1.1$ and $24.0 \pm 1.4$ years respectively. First year students have scored $40.6 \pm 23.8$ while final year students scored $41.5 \pm 20.8$ mean in the BEES measuring emotional empathy score. However, this difference was not statistically significant $(t=-0.30, d f=231, P$-value $>0.05)$. Final year students had significantly higher mean cognitive empathy score $(17.8 \pm 4.5)$ than first year students $(14.4 \pm 4.8)[\beta=2.7,95 \% \mathrm{Cl}(1.20,4.13)]$. Males had scored lower cognitive $[\beta=-2.5,95 \% \mathrm{Cl}(-4.37,-0.66)]$ and emotional empathy $[\beta=-12.0,95 \% \mathrm{Cl}(-21.66,-5.46)]$.

Conclusions: Low emotional (BEES) and cognitive empathy sores were found in first year and final year students of Jimma University could have implications on the medical education curricula. Medical education targeted at enhancing emotional empathy and increasing cognitive empathy is required by segmenting with gender for effective physician-patient interaction. The influence of empathy on clinical competence should be studied using more rigorous design.

Keywords: Cognitive empathy, Emotional empathy, Medical education, Jimma University

\footnotetext{
* Correspondence: grm_sht@yahoo.com

${ }^{\dagger}$ Equal contributors

${ }^{2}$ Department of Health Education and Behavioral Sciences, Jimma University, Jimma, Ethiopia

Full list of author information is available at the end of the article
} 


\section{Background}

It was in 1977 in Australia when empathy in medical students was measured for the first time [1]. Since then, plenty of investigations into empathy at medical schools all over the world followed [2-8], so far with no consideration of the continent of Africa. Empathy has been described as a concept involving cognitive as well as emotional domains [9]. The cognitive domain of empathy involves the ability to understand another person's inner experiences and feelings and a capability to view the outside world from the other person's perspective [10]. Such a cognitive component is also amenable to training and, thus, medical schools can play a positive role in the development of students' understanding about empathy [4].

The emotional domain involves the capacity to enter into or join the experiences and feelings of another person $[10,11]$. The emotional relationships that elicit emotional response are conceptually more relevant to sympathy than to empathy [12]. Because sympathy, if excessive, could interfere with objectivity in diagnosis and treatment $[11,13]$, "compassionate detachment" has been used to describe the physician's empathetic concern for the patient while keeping sympathy at a reasonable distance to maintain an emotional balance $[13,14]$. Hence, an "emotional distance" would be desirable to avoid bursts of emotions that might interfere with clinical neutrality and personal durability [15].

Main findings of studies recorded a decline in empathy during medical school proceedings [3-5,16-22], a higher empathy level in females compared to males $[4,5,7,16,19,21-26]$, and a relation between the students' choice of the future medical specialization and their empathy level scores $[4,22,26,27]$. The disturbing possibility is that medical education might be injuring instead of nurturing empathy [28]

There is general consent that empathy is crucial for the physician-patient relationship and thus an important issue in medical education. Empathy represents the "touch" in modern medicine, at present ill-reputed as "high tech, low touch" [29]. As the theoretical constructs of empathy are complex, physicians' appropriate empathy is still under discussion. Jodi Halpern suggested an answer to the question "What is clinical empathy?" considering the attention to the patient as the focus of the physicians' task, not seeing any necessity of experiencing vicariously their patients' emotions [30]. Depending upon developmental, experiential, social, educational, and other endogenous and exogenous factors, one group may possess more or less empathy than another group [31].

So far, there is no study of empathy among medical students in Ethiopia; taking into account possible cultural influences on medical students' empathy or other empathy-influencing factors like socio-demographic and personal characteristics. In addition to experiences in clinical practice, since medical students in the University take courses on behavioral sciences, we expect final year students to have less emotional empathy and higher cognitive empathy than first year students. Hence, this study was intended to assess whether empathy increases with medical training and identify the socio-demographic background of medical students influencing their empathy level

\section{Methods}

Comparative cross-sectional study among 131 first year and 106 final year (fifth year) medical students was conducted in Jimma University, Ethiopia on academic year 2010/11. Jimma University is found in Jimma city located $350 \mathrm{~km}$ southwest of Addis Ababa, Ethiopia. There were about 1000 medical students in total, the number of first-year students being around 210 and final year students around 150. Assuming small to medium effect sizes (Cohen's d $=0.4$ ) and a power of $80 \%$, a sample size of 100 students per group was envisaged. In the end, 131 first year and 106 final year medical students were included in the study. The study subjects were selected using simple random sampling technique from the list of the students.

Two different self administered survey instruments were used to measure the students' empathy. The Balanced Emotional Empathy Scale (BEES) was used for the measurement of "heart-reading", i.e. emotional empathy [32]. The BEES is an instrument consisting of 15 positively and 15 negatively worded items that measure emotional responses to fictive situations and particular life events (examples of the items include: "Unhappy movie endings haunt me for hours afterward, I cannot feel much sorrow for those who are responsible for their own misery"). The coefficient alpha internal consistency of the BEES is 0.87 . The questions attempt to probe the extent to which the respondent is able to feel the other's suffering or take pleasure in their happiness. Study subjects report the degree of their agreement or disagreement for each of the 30 items using a 9-point Likert-scale. A higher total score represents a higher level of emotional empathy. The stated norm provided in the Manual for the BEES is $45 \pm 24$ [32].

As gaze perception plays a crucial role in the ability to reason about others' intentions and feelings [33], the Reading the Mind in the Eyes test (RME-R test) was used to evaluate "mind-reading", i.e. cognitive empathy. The RME$\mathrm{R}$ test consists of 36 photographs depicting just the eye regions. A rectangular area of approximately $5 \times 2$ in. delineated the eye region, encompassing the entire width of the face from midway up the nose to right above the brow. Four mental states accompanying each stimulus (one target word and three foils) were presented at each corner of 
the high resolution photograph. To reduce linguistic difficulties, the test had appended a detailed glossary where all adjectives were explained using synonyms and example sentences. A typical mean score is in the range 22-30. A mean score over 30 indicates a very accurate at decoding a person's facial expressions around the eyes. A score under 22 indicates a very low score of RME-R test [34].

Socio-demographic characteristics included questions about gender, age, year of education, ethnicity, country of birth, migration background, the people with whom the students grew up (mother/father/both/other), number of siblings, position (birth order) within siblings (eldest/sandwich/youngest), major life events during childhood (divorce/illness/death of parents), place of residence (at home with relatives/moved out), socio-economic status of parents, religion, active membership in a religious community, number of close relationships, involvement in online social networks like facebook, daily internet use, interest in a medical specialization (specialization with continuity of patient care such as internal medicine, psychiatry and pediatrics versus specialization with less interpersonal contact such as surgery, radiology and pathology) and a question about students' personal experience with psychiatric or psychotherapeutic treatment.

To deal with missing values in the self-rating scale BEES, imputation by the individual mean of the observed items was applied separately for the positively and the negatively worded items, in case the respective number of missing values did not exceed 5 (i.e. 33\%). Otherwise the questionnaire was treated as insufficient and was excluded from the analyses. In the RME-R test, missing answers were treated as "the participant did not recognize the emotion". However, if more than the half of the RME-R questionnaire was empty, this was interpreted as insufficient motivation to complete the test, which was therefore excluded from the analyses. Apart from descriptive statistics, $t$-test for empathy and RME-R scores were performed to check for mean difference between the two groups of students. Pearson correlations between the BEES and the RME-R were also calculated. A linear regression was computed between the empathy scores and socio-demographic and other background characteristics using the enter method. A significance level of $5 \%$ was used. All analyses were performed using SPSS version 16.

Ethical approval for the study was granted by the Ethics Review Board of Jimma University, college of public health and Medical Sciences. After a brief explanation of the study, a written consent was obtained from each participant.

\section{Results}

\section{Characteristics of the study participants}

Out of the total 237 students, 207 (87.3\%) were males. The mean ages of first year and final year students were
$19.3 \pm 1.1$ and $24.0 \pm 1.4$ years respectively. The combined mean age was $21.4 \pm 2.7$ years. Majority of them 119(50.2\%) were Oromo in ethnicity and Christian religion followers, $177(74.7 \%)$. Most of the students 195 $(82.3 \%)$ were living with both of their parents. More than half $118(52.4 \%)$ of the students were neither the youngest nor the eldest child in their family (i.e. are sandwich) and were living with their family or relatives; 131(58.2\%). A large proportion of the students 156 (67.2\%) perceived themselves as active members of their religion. Only 77(32.9\%) of them have ever faced major life events (divorce/illness/death of parents) during their childhood and 7(3.0\%) had a history of migration. Majority 141(60.3) of the students were currently using online social media like facebook as a social media. Small proportion 27(12.3\%) have never used internet. Most of them 147(63.4\%) were interested in specialization with continuity of patient care (i.e. specializations which have more interactions with patients). Sixteen (6.8\%) of the students had a history of psychiatric treatment (Table 1 ).

\section{Emotional empathy}

First year students had mean BEES score of $39.0 \pm 22.3$ for male and $51.8 \pm 30.6$ for female. Final year students had mean BEES score of $39.9 \pm 20.0$ for male and $51.5 \pm 23.5$ for female. Male students had statistically significant lower mean BEES score $(t=-2.81, d f=233$, $\mathrm{p}$-value $<0.05$ ). First year as well as final year students who were using social media like facebook has scored higher mean emotional empathy scores $(45.2 \pm 24.1$ and $42.6 \pm 21.3)$ respectively. Use of social media like facebook had significant statistical association $(t=2.20$, $\mathrm{df}=195$, P-value $<0.05$ ) with mean emotional empathy score. Generally, first year students have scored $40.6 \pm 23.8$ while final year students have scored $41.5 \pm 20.8$ mean emotional empathy (Table 2). The median emotional empathy score was also almost similar for both groups of students (Figure 1). Also, there was no statistically significant difference between first year and final year medical students on the mean emotional empathy score $(t=-0.30, \mathrm{df}=231$, P-value $>0.05)$.

The regression analysis has indicated that first year male students had less emotional empathy $[\beta=-13.7$, $95 \%$ CI $(-27.22,-0.19)]$ than first year female students. There was no significant statistical difference in emotional empathy score with the remaining socio-demographic characteristics of first year students. There was no significant statistical emotional empathy score difference with any of the socio-demographic characteristics among final year students. The overall regression analysis indicated that male compared with female $[\beta=-12.0,95 \% \mathrm{CI}(-21.66,-5.46)]$ and those who have not decided about future specialization interest area 
Table 1 Socio-demographic and background characteristics of first year and final year medical students of Jimma University; Ethiopia, 2011

\begin{tabular}{|c|c|c|c|c|}
\hline \multirow[t]{2}{*}{ Variable } & & \multicolumn{2}{|c|}{ Year № (\%) } & \multirow[t]{2}{*}{ P-value } \\
\hline & & First year $\left(n_{1}=131\right)$ & Final year $\left(n_{2}=106\right)$ & \\
\hline \multirow[t]{2}{*}{ Sex } & Male & $115(87.8)$ & $92(86.8)$ & 0.91 \\
\hline & Female & $16(12.2)$ & 14(13.2) & \\
\hline \multirow[t]{3}{*}{ Ethnicity } & Oromo & $52(39.7)$ & $67(63.2)$ & 0.01 \\
\hline & Amhara & $46(35.1)$ & $28(26.4)$ & \\
\hline & Others & $33(25.4)$ & $11(10.4)$ & \\
\hline \multirow[t]{4}{*}{ Grow up with } & Mother & $8(6.1)$ & $11(10.4)$ & 0.01 \\
\hline & Father & $3(2.3)$ & $4(3.8)$ & \\
\hline & Both & $108(82.4)$ & $87(82.1)$ & \\
\hline & Other & $12(9.2)$ & $4(3.8)$ & \\
\hline \multirow[t]{3}{*}{ Position in the family } & Eldest & $32(26.4)$ & $33(31.7)$ & 0.18 \\
\hline & sandwich & $61(50.8)$ & $57(54.8)$ & \\
\hline & youngest & $28(23.3)$ & $14(13.5)$ & \\
\hline \multirow[t]{2}{*}{ Place of living } & At home with family or relatives & $71(57.7)$ & $60(58.8)$ & 0.68 \\
\hline & Not at home & $52(42.3)$ & $42(41.2)$ & \\
\hline \multirow[t]{3}{*}{ Working status of mother } & working & 49(39.8) & $52(53.1)$ & 0.30 \\
\hline & housewife & $66(53.7)$ & $40(40.8)$ & \\
\hline & out of work & $8(6.5)$ & $6(6.1)$ & \\
\hline \multirow[t]{3}{*}{ Working status of father } & working & $101(82.8)$ & $84(87.5)$ & 0.01 \\
\hline & houseman & $6(4.9)$ & $8(8.3)$ & \\
\hline & out of work & $15(12.3)$ & $4(4.2)$ & \\
\hline \multirow[t]{3}{*}{ Religion } & Christian & 101(77.1) & 76(71.7) & 0.32 \\
\hline & Muslim & $28(21.4)$ & $29(27.4)$ & \\
\hline & Other & $2(1.5)$ & $1(0.9)$ & \\
\hline \multirow[t]{2}{*}{ Religion active member } & yes & $95(73.6)$ & $61(59.2)$ & 0.06 \\
\hline & No & $34(26.4)$ & $42(40.8)$ & \\
\hline \multirow[t]{2}{*}{ Major life event } & yes & $45(34.9)$ & $32(30.5)$ & 0.30 \\
\hline & no & $84(65.1)$ & $73(69.5)$ & \\
\hline \multirow[t]{2}{*}{ Online social media like facebook } & yes & $51(39.8)$ & $90(84.9)$ & $<0.001$ \\
\hline & No & $77(60.2)$ & $16(15.1)$ & \\
\hline \multirow[t]{2}{*}{ Migration history } & Yes & $1(0.8)$ & $6(5.7)$ & 0.09 \\
\hline & No & 129(99.2) & $100(94.3)$ & \\
\hline \multirow[t]{3}{*}{ Daily internet time } & less than $1 \mathrm{~h}$ & $90(77.6)$ & $74(71.2)$ & 0.04 \\
\hline & more than $1 \mathrm{~h}$ & $11(9.5)$ & $18(17.3)$ & \\
\hline & no internet & $15(12.9)$ & $12(11.5)$ & \\
\hline \multirow[t]{3}{*}{ Interested in } & continuity of patient care & $75(58.6)$ & $72(69.2)$ & 0.47 \\
\hline & less interpersonal contact & $31(24.2)$ & $18(17.3)$ & \\
\hline & no idea & $22(17.2)$ & $14(13.5)$ & \\
\hline \multirow[t]{2}{*}{ History of Psychiatric treatment } & yes & $7(5.5)$ & $9(8.5)$ & 0.65 \\
\hline & No & $121(94.5)$ & $97(91.5)$ & \\
\hline
\end{tabular}

*For some of the variables the individual sum is not equal to the total sample size since there are missing values. 
Table 2 Mean and standard deviation score of BEES of first year and final year medical students of Jimma University; Ethiopia, 2011

\begin{tabular}{|c|c|c|c|c|}
\hline \multirow[t]{2}{*}{ Variable } & & \multicolumn{2}{|c|}{ Mean BEES (SD) } & \multirow[t]{2}{*}{ P-value } \\
\hline & & First year $\left(n_{1}=131\right)$ & Final year $\left(n_{2}=106\right)$ & \\
\hline \multirow[t]{2}{*}{ Sex } & Male & $39.0(22.3)$ & $39.9(20.0)$ & 0.01 \\
\hline & Female & $51.8(30.6)$ & $51.5(23.5)$ & \\
\hline \multirow[t]{3}{*}{ Ethnicity } & Oromo & $38.4(23.2)$ & $39.3(21.8)$ & 0.26 \\
\hline & Amhara & $38.9(25.3)$ & $46.1(20.2)$ & \\
\hline & Others & $46.3(22.2)$ & $43.0(14.1)$ & \\
\hline \multirow[t]{4}{*}{ Grow up with } & Mother & $37.3(17.6)$ & $38.6(20.3)$ & 0.72 \\
\hline & Father & $37.3(2.5)$ & $54.0(31.6)$ & \\
\hline & Both & $40.1(24.3)$ & $41.4(20.6)$ & \\
\hline & Other & $47.6(25.3)$ & $39.3(18.7)$ & \\
\hline \multirow[t]{3}{*}{ Position in the family } & Eldest & $48.2(18.8)$ & $38.0(23.5)$ & 0.24 \\
\hline & sandwich & $41.3(24.0)$ & 43.3(18.6) & \\
\hline & youngest & $32.2(27.8)$ & $42.8(23.2)$ & \\
\hline \multirow[t]{2}{*}{ Place of living } & At home with family or relatives & $41.9(24.1)$ & $43.2(19.1)$ & 0.16 \\
\hline & Not at home & $39.0(24.0)$ & $37.5(22.6)$ & \\
\hline \multirow[t]{3}{*}{ Working status of mother } & working & $39.6(26.7)$ & $42.3(20.9)$ & 0.86 \\
\hline & housewife & $41.4(21.5)$ & $39.3(19.4)$ & \\
\hline & out of work & $37.8(26.4)$ & $37.3(15.2)$ & \\
\hline \multirow[t]{3}{*}{ Working status of father } & working & $40.5(24.6)$ & $40.1(20.8)$ & 0.98 \\
\hline & houseman & $29.5(17.6)$ & 49.4(30.9) & \\
\hline & out of work & $41.1(18.8)$ & $39.0(8.0)$ & \\
\hline \multirow[t]{3}{*}{ Religion } & Christian & $39.7(24.6)$ & $40.8(20.0)$ & 0.54 \\
\hline & Muslim & $42.9(21.3)$ & $43.2(23.3)$ & \\
\hline & Other & $53.5(9.2)$ & $41.0(0.0)$ & \\
\hline \multirow[t]{2}{*}{ Religion active member } & Yes & $44.0(23.1)$ & $40.2(20.1)$ & 0.18 \\
\hline & No & $32.7(23.5)$ & $42.8(22.3)$ & \\
\hline \multirow[t]{2}{*}{ Major life event } & Yes & $44.8(22.4)$ & $39.9(21.9)$ & 0.32 \\
\hline & No & $37.8(24.1)$ & $42.0(20.5)$ & \\
\hline \multirow[t]{2}{*}{ Online social media like facebook } & Yes & $45.2(24.1)$ & $42.6(21.3)$ & 0.03 \\
\hline & No & $37.2(22.8)$ & $35.2(16.9)$ & \\
\hline \multirow[t]{2}{*}{ Migration history } & Yes & $98.0(0.0)$ & $33.2(28.0)$ & 0.87 \\
\hline & No & $40.2(23.3)$ & $42.0(20.3)$ & \\
\hline \multirow[t]{3}{*}{ Daily internet time } & less than $1 \mathrm{~h}$ & $39.7(24.1)$ & $42.2(22.5)$ & 0.85 \\
\hline & more than $1 \mathrm{~h}$ & $36.0(27.8)$ & $40.2(17.8)$ & \\
\hline & no internet & 39.6(19.5) & $37.7(15.2)$ & \\
\hline \multirow[t]{3}{*}{ Interested in } & Continuity of patient care & $41.8(23.9)$ & $43.0(20.4)$ & 0.10 \\
\hline & less interpersonal contact & $40.8(24.6)$ & $43.9(20.0)$ & \\
\hline & no idea & $32.7(20.0)$ & $35.1(21.1)$ & \\
\hline \multirow[t]{2}{*}{ History of Psychiatric treatment } & Yes & $26.3(22.9)$ & $37.9(19.4)$ & 0.12 \\
\hline & No & $41.7(23.7)$ & $41.8(21.0)$ & \\
\hline Total BEES mean (SD) & $40.6(23.8)$ & $41.5(20.8)$ & $41.1(22.4)$ & 0.81 \\
\hline
\end{tabular}




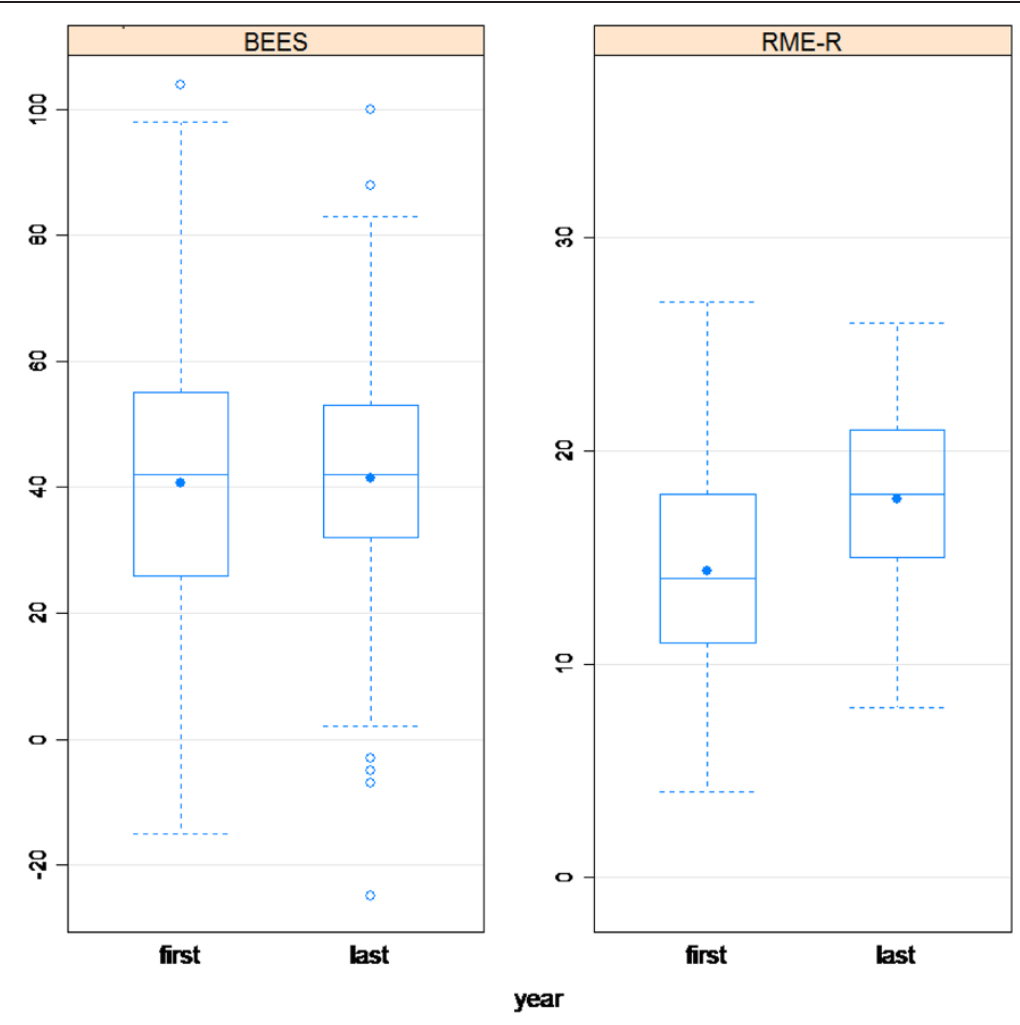

Figure 1 Box plot showing BEES and RME-R scores of first year and final year students of Jimma University; Ethiopia, 2011.

compared with who decided $[\beta=-11.12,95 \%$ CI $(-20.91$, -1.34 )] had less emotional empathy. Students who were using social media like facebook had significantly higher emotional empathy score than non-users $[\beta=11.8,95 \%$ CI $(4.05,19.43)]$ (Table 3).

\section{Cognitive empathy}

Male students have scored lower mean cognitive empathy score than females both in first year and final year (14.2 \pm 4.7 for males Vs $16.3 \pm 4.8$ for females) and (17.2 \pm 4.3 for males Vs $21.2 \pm 4.0$ for females) respectively. Sex and cognitive empathy had significant statistical association $(t=-3.12, \mathrm{df}=37, \mathrm{P}$-value $<0.05)$. Age of the students had statistically significant positive correlation with cognitive empathy score (Pearson correlation $(r)=0.26, \quad P$-value $<0.05)$. The number of brothers and sisters students have had statistically significant negative correlation with cognitive empathy score of the students (Pearson correlation $(r)=-0.26$, P-value $<0.05)$.

Both class of students living at home with family or relatives had higher mean cognitive empathy scores $(14.9 \pm 4.8$ for first year and $18.6 \pm 4.6$ for final year) than those who lived alone. Whether students are living at home or not had significant statistical association $(t=2.25, d f=207, P$-value $<0.05)$. Both first year and final year students who were using social media like facebook have scored higher mean cognitive empathy $(15.8 \pm 4.4$ for first year and $17.8 \pm 4.5$ for final year students). Use of social media like facebook $(t=4.45, \mathrm{df}=$ 177 , P-value $<0.05$ ) and mothers socioeconomic condition $(\mathrm{t}=3.15, \mathrm{df}=199, \mathrm{P}$-value $<0.05)$ had significant statistical association. Final year students had (17.8 \pm 4.5$)$ mean cognitive empathy score and first year students had (14.4 \pm 4.8$)$ mean cognitive empathy (Table 3). Year of study and mean cognitive empathy score had significant statistical association $(\mathrm{t}=-5.50, \mathrm{df}=226, \mathrm{P}$-value $<0.05$ ) (Table 4). The median cognitive empathy score also has shown that final year students had higher cognitive empathy score than first year students (Figure 1). There was no statistically significant difference among first year students with all remaining socio-demographic characteristics. Final year male students have scored statistically lower cognitive empathy $[\beta=-3.9,95 \% \mathrm{CI}(6.49$, $-1.23)$ ] compared with final year female students. Based on the overall regression analysis, males had significantly lower $[\beta=-2.5,95 \% \mathrm{CI}(-4.37,-0.66)]$ cognitive empathy score than female students. As the number of brothers and sisters the students had increased, the cognitive empathy scored decreased significantly $[\beta=-0.4,95 \% \mathrm{CI}$ $(-0.65,-0.20)]$. Students who were using social media like facebook had significantly higher cognitive empathy 
Table 3 Predictors of BEES score of first year and final year medical students of Jimma University; Ethiopia, 2011

\begin{tabular}{|c|c|c|c|c|}
\hline \multirow[t]{2}{*}{ Variables } & \multirow[t]{2}{*}{$\beta$} & \multirow[t]{2}{*}{ Std. Error P-value } & \multicolumn{2}{|c|}{ 95\% Confidence Interval for $\beta$} \\
\hline & & & Lower Bound & Upper Bound \\
\hline Number of brothers and sisters & -0.84 & 0.58 & -1.99 & 0.31 \\
\hline \multicolumn{5}{|l|}{ Religion (Reference $=$ Muslim) } \\
\hline Christian & -3.62 & 3.91 & -11.34 & 4.10 \\
\hline Other & 5.38 & 13.83 & -21.88 & 32.65 \\
\hline \multicolumn{5}{|l|}{ Sex (Reference $=$ female) } \\
\hline Male & -12.03 & 4.89 & -21.66 & -2.39 \\
\hline \multicolumn{5}{|l|}{ Year of study (Reference $=$ first) } \\
\hline Second year & 5.72 & 7.63 & -9.32 & 20.76 \\
\hline \multicolumn{5}{|l|}{ Migration history (reference $=$ yes) } \\
\hline No & 6.05 & 9.05 & -11.80 & 23.90 \\
\hline \multicolumn{5}{|c|}{ Grown up with (Reference $=$ Mother) } \\
\hline Father & 0.35 & 2.14 & 0.16 & 0.87 \\
\hline Both & 1.33 & 1.20 & 1.10 & 0.27 \\
\hline Other & -0.10 & 1.71 & -0.06 & 0.95 \\
\hline \multicolumn{5}{|c|}{ Position in the family (Reference $=$ Eldest) } \\
\hline Sandwich & 5.76 & 3.57 & -1.29 & 12.81 \\
\hline Youngest & -3.76 & 4.65 & -12.93 & 5.42 \\
\hline \multicolumn{5}{|l|}{ Major life event (reference = No) } \\
\hline Yes & 2.65 & 3.65 & -4.55 & 9.85 \\
\hline \multicolumn{5}{|l|}{ Mothers job $($ reference $=$ working $)$} \\
\hline Housewife & 2.23 & 3.37 & -4.42 & 8.88 \\
\hline Out of work & 5.03 & 7.56 & -9.87 & 19.93 \\
\hline \multicolumn{5}{|l|}{ Fathers job (reference = working) } \\
\hline Houseman & 5.49 & 6.40 & -7.14 & 18.11 \\
\hline Out of work & -4.12 & 9.55 & -22.95 & 14.70 \\
\hline \multicolumn{5}{|c|}{ Religion active member $($ reference $=\mathbf{~ N o}$ ) } \\
\hline Yes & 0.620 & 3.31 & -5.92 & 7.16 \\
\hline \multicolumn{5}{|c|}{ Use of social media like facebook (reference = No) } \\
\hline Yes & 11.75 & 3.90 & 4.05 & 19.43 \\
\hline \multicolumn{5}{|c|}{ Daily internet use (reference $=$ no internet $)$} \\
\hline Less than $1 \mathrm{~h}$ & -7.23 & 4.43 & -15.97 & 1.51 \\
\hline more than $1 \mathrm{~h}$ & -10.81 & 6.15 & -22.95 & 1.33 \\
\hline \multicolumn{5}{|c|}{ Interested in (reference = less interpersonal contact) } \\
\hline in patient care & 1.97 & 3.75 & -5.43 & 9.36 \\
\hline No idea & -11.12 & 4.96 & -20.91 & -1.34 \\
\hline \multicolumn{5}{|c|}{ History of psychiatric treatment $($ reference $=\mathrm{No}$ ) } \\
\hline Yes & -5.82 & 5.89 & -17.44 & 5.80 \\
\hline$R^{2}=0.18$ & & & & \\
\hline
\end{tabular}


Table 4 Mean and standard deviation score of RME-E of first year and final year medical students of Jimma University; Ethiopia, 2011

\begin{tabular}{|c|c|c|c|c|}
\hline \multirow[t]{2}{*}{ Variable } & & \multicolumn{2}{|c|}{ Mean RME-R (SD) } & \multirow[t]{2}{*}{ P-value } \\
\hline & & First year $\left(n_{1}=131\right)$ & Final year $\left(n_{2}=106\right)$ & \\
\hline \multirow[t]{2}{*}{ Sex } & Male & $14.2(4.7)$ & $17.2(4.3)$ & $<0.01$ \\
\hline & Female & 16.3(4.8) & $21.2(4.0)$ & \\
\hline \multirow[t]{3}{*}{ Ethnicity } & Oromo & 13.6(4.6) & $17.0(4.4)$ & 0.31 \\
\hline & Amhara & $14.7(4.8)$ & 19.5(4.7) & \\
\hline & Others & 15.3(5.1) & 18.4(3.2) & \\
\hline \multirow[t]{4}{*}{ Grow up with } & Mother & 13.6(5.2) & 17.6(4.3) & 0.35 \\
\hline & Father & $12.0(2.0)$ & $22.8(4.6)$ & \\
\hline & Both & $14.7(4.8)$ & 17.6(4.4) & \\
\hline & Other & 13.7(5.3) & 16.3(5.0) & \\
\hline \multirow[t]{3}{*}{ Position in the family } & Eldest & 16.1(5.0) & $17.9(5.1)$ & 0.22 \\
\hline & Sandwich & $14.0(4.4)$ & $17.8(4.1)$ & \\
\hline & Youngest & 14.1(5.3) & $17.5(5.0)$ & \\
\hline \multirow[t]{2}{*}{ Place of living } & At home with family or relatives & $14.9(4.8)$ & 18.6(4.6) & 0.03 \\
\hline & Not at home & $14.1(4.7)$ & 16.3(4.0) & \\
\hline \multirow[t]{3}{*}{ Working status of mother } & Working & $15.2(5.3)$ & 18.5(4.1) & $<0.01$ \\
\hline & Housewife & $13.9(4.1)$ & 16.5(4.6) & \\
\hline & out of work & 17.3(5.6) & $20.8(4.4)$ & \\
\hline \multirow[t]{3}{*}{ Working status of father } & Working & $14.5(4.8)$ & $17.6(4.3)$ & 0.80 \\
\hline & Houseman & $11.7(2.9)$ & $17.6(4.9)$ & \\
\hline & out of work & 15.6(4.3) & 20.8(4.0) & \\
\hline \multirow[t]{3}{*}{ Religion } & Christian & 14.4(4.8) & $17.8(4.5)$ & 0.98 \\
\hline & Muslim & 14.6(5.0) & $17.6(4.7)$ & \\
\hline & Other & $14.5(0.7)$ & $17.0(0.0)$ & \\
\hline \multirow[t]{2}{*}{ Religion active member } & Yes & 14.3(4.5) & $17.9(4.7)$ & \\
\hline & No & $15.2(5.2)$ & $17.5(4.2)$ & \\
\hline \multirow[t]{2}{*}{ Major life event } & Yes & 14.8(5.1) & 17.3(5.1) & 0.66 \\
\hline & No & $14.2(4.7)$ & 18.1(4.1) & \\
\hline \multirow[t]{2}{*}{ Online social media like facebook } & Yes & $15.8(4.4)$ & $17.8(4.5)$ & $<0.001$ \\
\hline & No & $13.5(4.9)$ & $17.6(4.3)$ & \\
\hline \multirow[t]{2}{*}{ Migration history } & Yes & $14.8(5.1)$ & 17.3(5.1) & 0.86 \\
\hline & No & $14.2(4.7)$ & 18.1(4.1) & \\
\hline \multirow[t]{3}{*}{ Daily internet time } & less than $1 \mathrm{~h}$ & $14.4(5.1)$ & $17.8(4.8)$ & 0.35 \\
\hline & more than $1 \mathrm{~h}$ & 14.8(4.9) & 18.2(3.9) & \\
\hline & no internet & 14.4(3.7) & 16.8(3.6) & \\
\hline \multirow[t]{3}{*}{ Interested in } & continuity of patient care & 13.9(4.9) & 18.2(4.2) & 0.13 \\
\hline & less interpersonal contact & $16.2(4.7)$ & 18.4(4.9) & \\
\hline & no idea & 14.0(4.3) & $16.14(4.1)$ & \\
\hline \multirow[t]{2}{*}{ History of Psychiatric treatment } & Yes & 13.6(7.8) & 14.4(4.6) & 0.11 \\
\hline & No & 14.6(4.6) & 18.1(4.4) & \\
\hline Total BEES mean (SD) & & 14.4(4.8) & $17.8(4.5)$ & $<0.001$ \\
\hline
\end{tabular}


Table 5 Predictors of RME-E score of first year and final year medical students of Jimma University; Ethiopia, 2011

\begin{tabular}{|c|c|c|c|c|}
\hline \multirow[t]{2}{*}{ Variables } & \multirow[t]{2}{*}{$\beta$} & \multirow[t]{2}{*}{ Std. Error } & \multicolumn{2}{|c|}{ 95\% Confidence Interval for $\beta$} \\
\hline & & & Lower Bound & Upper Bound \\
\hline Number of brothers and sisters & -0.43 & 0.12 & -0.65 & -0.20 \\
\hline \multicolumn{5}{|l|}{ Religion (Reference = Muslim) } \\
\hline Christian & -0.54 & 0.78 & -2.08 & 1.00 \\
\hline Other & -0.80 & 2.73 & -6.19 & 4.59 \\
\hline \multicolumn{5}{|l|}{ Sex (Reference = female) } \\
\hline Male & -2.51 & 0.94 & -4.37 & -0.66 \\
\hline \multicolumn{5}{|l|}{ Year of study (Reference $=$ first) } \\
\hline Second year & 2.66 & 0.74 & 1.20 & 4.13 \\
\hline \multicolumn{5}{|l|}{ Migration history (reference $=$ yes) } \\
\hline No & 2.83 & 1.81 & -0.75 & 6.40 \\
\hline \multicolumn{5}{|l|}{ Grown up with (Reference $=$ Mother) } \\
\hline Father & 0.45 & 2.13 & -3.76 & 4.65 \\
\hline Both & 1.32 & 1.20 & -1.05 & 3.69 \\
\hline Other & 0.03 & 1.69 & -3.30 & 3.36 \\
\hline \multicolumn{5}{|c|}{ Position in the family (Reference = Eldest) } \\
\hline Sandwich & 0.44 & 0.72 & -0.98 & 1.85 \\
\hline Youngest & -1.13 & 0.91 & -2.93 & 0.68 \\
\hline \multicolumn{5}{|l|}{ Major life event (reference = No) } \\
\hline Yes & 0.41 & 0.73 & -1.02 & 1.84 \\
\hline \multicolumn{5}{|l|}{ Mothers job (reference = working) } \\
\hline Housewife & -0.80 & 0.68 & -2.14 & 0.53 \\
\hline Out of work & 3.17 & 1.51 & 0.19 & 6.15 \\
\hline \multicolumn{5}{|l|}{ Fathers job (reference = working) } \\
\hline Houseman & -0.04 & 1.28 & -2.56 & 2.48 \\
\hline Out of work & -1.19 & 1.90 & -4.94 & 2.56 \\
\hline \multicolumn{5}{|c|}{ Religion active member (reference $=\mathbf{N o}$ ) } \\
\hline Yes & -0.79 & 0.67 & -2.10 & 0.53 \\
\hline \multicolumn{5}{|c|}{ Use of social media like facebook (reference = No) } \\
\hline Yes & 1.93 & 0.78 & 0.40 & 3.46 \\
\hline \multicolumn{5}{|c|}{ Daily internet use (reference = no internet) } \\
\hline Less than $1 \mathrm{~h}$ & -0.58 & 0.88 & -2.31 & 1.15 \\
\hline more than $1 \mathrm{~h}$ & -0.33 & 1.25 & -2.79 & 2.13 \\
\hline \multicolumn{5}{|c|}{ Interested in (reference = less interpersonal contact) } \\
\hline in patient care & -0.46 & 0.75 & -1.95 & 1.02 \\
\hline No idea & -1.68 & 0.99 & -3.63 & 0.27 \\
\hline \multicolumn{5}{|c|}{ History of psychiatric treatment $($ reference $=\mathrm{No}$ ) } \\
\hline Yes & -2.16 & 1.18 & -4.48 & 0.16 \\
\hline$R^{2}=0.33$ & & & & \\
\hline
\end{tabular}


score than non-users $[\beta=1.9,95 \% \mathrm{CI}(0.40,3.46)]$. Final year students have scored significantly higher $[\beta=2.7$, $95 \%$ CI $(1.20,4.13)]$ cognitive empathy score than first year students (Table 5).

\section{Reliability of BEES and RME-R}

The BEES and RME-R measures had acceptable reliability with Cronbach's alpha of 0.72 and 0.70 respectively. There was weak positive correlation (Pearson correlation $(r)=0.29)$ between emotional and cognitive empathy measures in this study.

\section{Discussion}

Our study has found that there was no significant difference in emotional empathy between first year and final year medical students. But there was significantly higher cognitive empathy among final year students as compared to first year students. Sex was a predictor variable for both cognitive and emotional empathy. In addition, future specialization interest area and being active user of online social media like facebook were also predictor variables for emotional empathy among medical students.

Compared with the stated norms of empathy used by an instruments used for this study [32,34], there was lower mean emotional and cognitive empathy scores among Ethiopian first year and final year medical students. Preserving the low emotional empathy at final year similar to first year, may be a positive result of the students training and experience through clinical years of the students. It has been stated that 'emotional relationships that elicit emotional response are conceptually more relevant to sympathy than to empathy' [12].

Both cross-sectional and longitudinal studies have indicated that females have generally more emotional empathy than males $[16,19,21-26]$. According to psychoanalytic and evolutionary theory of parental investment, women are believed to develop greater care-giving attitudes toward their offspring than men [35] and these caring characteristics can be associated with high emotional empathy. Even the norm for empathy measure has set much higher empathy score of female than male [32]. As it has been indicated with all the above findings, male students had much lower emotional empathy scores in this particular study. But gender based comparative study is required with representative proportions to make inference in this regard.

Even though we found that final year students have scored statistically higher cognitive empathy score than first year students, practically the difference was below the standard; according to RME- $\mathrm{R}$ test, a mean score under 22 indicates low score [34]. This may be related with the validity of the instruments used to the culture of the study participants (the instrument used was with a Caucasian faces in the photos). In any case, final year students had higher cognitive empathy score than first year students. Similar to emotional empathy score, this may be also associated with the training in medical education or experience during the clinical years. Similar studies have found contradicting findings in this regard; studies in Japan and Korea found the highest values for measures of empathy, by year of medical school, among senior medical students $[7,36]$ while another study in Iran did not find variations in empathy [37]. This difference might be attributed to the instruments used; since, in the Korean study they measured clinical empathy than general empathy.

Unlike that of emotional empathy, females have scored higher cognitive empathy than the males which may suggest that female may provide a better type of medical care [38-40] based on a better understanding of the patient's experiences and feelings (cognitive empathy). In a number of studies, a higher empathy level in females was found as compared to males [4,5,7,16,19,21-26].

Even though many findings of studies recorded a decline in empathy during medical school proceedings [3-5,16-22], in a normal circumstances we expect emotional empathy to decrease and cognitive empathy to increase as students progress through the years of medical school training. Our finding is also compatible with such a normal situation. The weak positive correlation between the two measures may be indicative of this explanation.

Previous studies found an association between the choice of medical students' future specialization and their empathy level scores $[4,22,26,27]$. In our study, we found that students who did not decide about future interest specialization area had low emotional empathy than who have decided which may be attributed to first year students may not be familiar about some of the medical specialization areas and were still in undecided situation.

Students who were using online social media like facebook had significantly higher both emotional and cognitive empathy score as non-users. There was no documented previous study on the effect of using social media like facebook on empathy. Hence, further study is required to give more explanations for such differences. Another issue that needs further study is why there was inverse relationship between cognitive empathy and the number of brothers and sisters students had.

There was no statistically significant difference with other socio-demographic variables in this study. This may be due to similarity of the different cultures with regard to empathy in Ethiopia. One of the strength of this study is we have tried to measure two dimensions of empathy and the method of data collection was self administered study so that there may be less social desirability bias. Since the study is of explanatory nature, 
it is not worth adjusting for multiple testing. The validity of the study may be limited by a cross-sectional rather than longitudinal design of the study. The relatively small sample size and the fact that both instruments of the emotional and cognitive empathy scales were not validated in Ethiopia are the main limitations of this study. Nonetheless, this is the first study in the area and we believe it will add valuable information to the existing knowledge gap.

\section{Conclusions}

Low emotional BEES score and cognitive empathy score (RME-R test score) was found in first year and final year students of Jimma University may imply that the medical school curricula should improve training in empathy skills. Females were more emotional to internalize the pains and also understand the feelings of others more easily from the eyes than males. Medical education targeted at enhancing appropriate emotional empathy and increasing cognitive empathy is required by segmenting with gender of the medical students for effective physician-patient interaction. To assure all these differences on patient care, the association between empathy and clinical competence should be studied with more rigorous designs. The issue of gender-based differences in medical care given by male and female medical students needs to be investigated. Culturally validated instrument is also required for further studies of empathy.

\section{Competing interests}

The authors declare that they have no competing interests.

\section{Acknowledgment}

We would like to thank Ayalkibet Jiru, Desta Thushune, Hailemarian Hailesilasiie, and Kinfe Tesfay for general support in Jimma. Special thanks go to the first and final year medical students in Jimma University. This study was supported by the Köhler foundation.

\section{Author details \\ ${ }^{1}$ Department of Psychiatry and Psychotherapy, Ludwig-Maximilians- University, Munich, Germany. ${ }^{2}$ Department of Health Education and Behavioral Sciences, Jimma University, Jimma, Ethiopia. ${ }^{3}$ Department of Psychiatry, Jimma University, Jimma, Ethiopia. ${ }^{4}$ Department of Surgery, Ludwig-Maximilians-University, Munich, Germany.}

\section{Authors' contributions}

MS was involved in the designed the study. SG was involved in the design of the study and collection of the data. SM was involved in the design of the study and analysis of the data. EG was involved in the collection, analysis of the data, drafting the manuscript and critically reviewing the manuscript. SD and MT were involved in design of the study, analysis of the data and critically reviewing the manuscript. All authors read and approved the final manuscript.

Received: 13 March 2012 Accepted: 24 May 2012

Published: 24 May 2012

\section{References}

1. Hornblow AR, Kidson MA, Jones KV: Measuring medical students' empathy: a validation study. Med Educ 1977, 11:7-12.

2. Pedersen R: Empirical research on empathy in medicine-a critical review. Patient Educ Couns 2009, 76:307-322.
3. Chen DC, Pahilan ME, Orlander JD: Comparing a self-administered measure of empathy with observed behavior among medical students. J Gen Intern Med 2010, 25:200-202.

4. Hojat M, Vergare MJ, Maxwell K, Brainard G, Herrine SK, Isenberg GA: The devil is in the third year: a longitudinal study of erosion of empathy in medical school. Acad Med 2009, 84:1182-1191.

5. Nunes P, Williams S, Sa B, Stevenson K: A study of empathy decline in students from five health disciplines during their first year of training. Int J Med Educ 2011, 2:12-17.

6. Rahimi-Madiseh M, Tavakol M, Dennick R, Nasiri J: Empathy in Iranian medical students: a preliminary psychometric analysis and differences by gender and year of medical school. Med Teach 2010, 32:e471-e478.

7. Roh MS, Hahm BJ, Lee DH, Suh DH: Evaluation of empathy among Korean medical students: a cross-sectional study using the Korean Version of the Jefferson Scale of Physician Empathy. Teach Learn Med 2010, 22:167-171.

8. Todres M, Tsimtsiou Z, Stephenson A, Jones R: The emotional intelligence of medical students: an exploratory cross-sectional study. Med Teach 2010, 32:e42-e48.

9. Davis MH: Empathy: a social psychological approach. Madison: Brown \& Benchmark Publishers; 1994.

10. Hojat M, Mangione S, Nasca TJ, Cohen MJM, Gonnella JS, Erdmann JB, Veloski JJ, Magee M: The Jefferson Scale of empathy: development and preliminary psychometric data. Educ Psychol Meas 2001, 61:349-365.

11. Aring CD: Sympathy and empathy. JAMA 1958, 167:448-452.

12. Stephan WG, Finlay KA: The role of empathy in improving inter- group relations. J Soc Issues 1999, 55:729-743.

13. Nightingale SD, Yarnold PR, Greenberg MS: Sympathy, empathy, and physician resource utilization. J Gen Intern Med 1991, 6:420-423.

14. Blumgart HL: Caring for the patient. N Engl J Med 1964, 270:449-456.

15. Jensen N: The empathic physician (letter). Arch Intern Med 1994, 154:108

16. Chen D, Lew R, Hershman W, Orlander J: A cross-sectional measurement of medical student empathy. J Gen Intern Med 2007, 22:1434-1438.

17. Diseker RA, Michielutte R: An analysis of empathy in medical students before and following clinical experience. J Med Educ 1981, 56:1004-1010.

18. Hojat M, Mangione S, Nasca TJ, Rattner S, Erdmann JB, Gonnella JS: An empirical study of decline in empathy in medical school. Med Educ 2004, 38:934-941.

19. Kataoka HU, Koide N, Ochi K, Hojat M, Gonnella JS: Measurement of empathy among Japanese medical students: psychometrics and score differences by gender and level of medical education. Acad Med 2009, 84:1192-1197.

20. Kliszcz J, Hebanowski M, Rembowski J: Level and dynamics of empathy in $\mathrm{I}$ and $\mathrm{VI}$ year students at the Medical Academy of Medicine in Gdansk. Pol Tyg Lek 1996, 51:55-58.

21. Kliszcz J, Hebanowski M, Rembowski J: Emotional and cognitive empathy in medical schools. Acad Med 1998, 73:541.

22. Newton BW, Barber L, Clardy J, Cleveland E, O'Sullivan P: Is there hardening of the heart during medical school? Acad Med 2008, 83:244-249.

23. Austin EJ, Evans P, Magnus B, O'Hanlon K: A preliminary study of empathy, emotional intelligence and examination performance in $\mathrm{MBChB}$ students. Med Educ 2007, 41:684-689.

24. Fernndez-Olano C, Montoya-Fernndez J, Salinas-Snchez AS: Impact of clinical interview training on the empathy level of medical students and medical residents. Med Teach 2008, 30:322-324.

25. Pawelczyk A, Pawelczyk T, Bielecki J: Differences in medical specialty choice and in personality factors among female and male medical students. Pol Merkur Lekarski 2007, 23:363-366.

26. Hojat M: Empathy in medical students as related to specialty interest, personality, and perceptions of mother and father. Personal Individ Differ 2005, 39:1205-1215.

27. Kliszcz J, Hebanowski M: Studies on empathy in doctors and medical students. Pol Merkur Lekarski 2001, 11:154-157.

28. Eunice M, Ana S, Patrício C, Manuel C: Empathy in senior year and first year medical students: a cross-sectional study. BMC Med Educ 2011, 11:52.

29. John F, Nan F: Physician-Patient Communication: A Lost Art? J Am Acad Orthop Surg 2002, 10:95-105.

30. Halpern J: What is clinical empathy? J Gen Intern Med 2003, 18:670-674

31. Hojat M, Gonnella J, Mangione S, Nasca T, Veloski J, Erdmann J, Callahan C, Magee M: Empathy in medical students as related to academic performance, clinical competence and gender. Med Educ 2002, 36:522-527. 
32. Mehrabian A: Manual for the Balanced Emotional Empathy Scale (BEES). 2000. Available from Albert Mehrabian, 1130 Alta Mesa Road, Monterey, CA 93940.

33. Simon Baron-Cohen S: Mind blindness: an essay on autism and theory of mind. Cambridge: MIT Press; 1995.

34. Baron-Cohen S, Wheelwright S, Hill J, Raste Y, Plumb I: The "Reading the Mind in the Eyes" Test revised version: a study with normal adults, and adults with Asperger syndrome or high-functioning autism. J Child Psychol Psychiatry 2001, 42:241-251.

35. Trivers RL: Parental Investment and Sexual Selection. In Sexual selection and the descent of man. Edited by Campbell B. Chicago: Aldine; 1972:136-799.

36. Kataoka H, Norio Koide N, Hojat M, Gonnella J: Measurement of empathy among japanese medical students: psychometrics and score differences by gender and level of medical education. Acad Med 2009, 84:1192-1197.

37. Rahimi-madiseh M, Tavakol M, Dennick R, Nasiri J: Empathy in Iranian medical students: a preliminary psychometric analysis and differences by gender and year of medical school. Med Teach 2010, 32:471-478.

38. Bertakis KD, Helms $L$, Callahan EJ, Azari R, Robbins JA: The influence of gender on physician practice style. Med Care 1995, 33:407-416.

39. Maheux B, Duford F, Beland F, Jaques A, Lavesque A: Female medical practitioners: more preventive and patient oriented? Med Care 1990 28:87-92.

40. Fruen M, Rothman A, Steiner J: Comparison of characteristics of male and female medical school applicants. J Med Educ 1974, 49:137-145.

doi:10.1186/1472-6920-12-34

Cite this article as: Dehning et al: Comparative cross-sectional study of empathy among first year and final year medical students in Jimma University, Ethiopia: Steady state of the heart and opening of the eyes. BMC Medical Education 2012 12:34.

\section{Submit your next manuscript to BioMed Central and take full advantage of:}

- Convenient online submission

- Thorough peer review

- No space constraints or color figure charges

- Immediate publication on acceptance

- Inclusion in PubMed, CAS, Scopus and Google Scholar

- Research which is freely available for redistribution 